\title{
Thermoelectric effects in strongly interacting quantum dot coupled to ferromagnetic leads
}

\author{
Mariusz Krawiec, Karol I. Wysokiński*, \\ Institute of Physics and Nanotechnology Center, M. Curie-Sktodowska University, Pl. M. Curie-Sktodowskiej 1, \\ PL 20-031 Lublin, Poland
}

\begin{abstract}
We study thermoelectric effects in Kondo correlated quantum dot coupled to ferromagnetic electrodes by calculating thermopower $\mathrm{S}$ in the Kondo regime as function of on-dot energy level and temperature. The system is represented by the Anderson model and the results agree well with those recently measured for a quantum dot coupled to nonmagnetic leads. For magnetic electrodes one observes marked dependence of S on the degree of their polarization.
\end{abstract}

Key words: quantum dot, thermopower, Kondo effect PACS: 75.20.Hr, 72.15.Qm, 72.25.-b, 73.23.Hk

Transport properties of artificial nanostructures with highly correlated electrons present fruitful research area. In particular the many body effects have been predicted [1] and later observed [2] in quantum dots coupled to external leads. Such structures allow study of various physical phenomena in well controlled conditions and geometries. Number of possibilities have already been tested experimentally resulting in discoveries of new effects [3].

In this contribution we are interested in the study of the voltage $V_{T}$ across quantum dot induced by the temperature gradient characterised by $\Delta T$. The ratio $\frac{V_{T}}{\Delta T}$ is a measure of the thermopower $S$. We consider single quantum dot with only one energy level coupled to two external ferromagnetic leads. The second electron on the dot experiences strong repulsion $U$ due to charging energy $e^{2} / 2 C$, where $C$ is the capacitance of the dot. We model the system by the $U=\infty$ single impurity Anderson Hamiltonian in the slave boson representation and adopt the calculation scheme and the approximations presented previously in [4].

\footnotetext{
* Corresponding author. Tel: +48815376236, fax:+4881 5376191

Email addresses: krawiec@kft.umcs.lublin.pl (Mariusz Krawiec), karol@tytan.umcs.lublin.pl (Karol I. Wysokiński).
}

$$
\begin{array}{r}
H=\sum_{\lambda \mathbf{k} \sigma} \epsilon_{\lambda \mathbf{k} \sigma} c_{\lambda \mathbf{k} \sigma}^{+} c_{\lambda \mathbf{k} \sigma}+\sum_{\sigma} E_{d} f_{\sigma}^{+} f_{\sigma} \\
+\sum_{\lambda \mathbf{k} \sigma}\left(V_{\lambda \mathbf{k} \sigma} c_{\lambda \mathbf{k} \sigma}^{+} b^{+} f_{\sigma}+\text { H.c. }\right),
\end{array}
$$

where $\lambda=L(R)$ denotes left (right) lead, $c_{\lambda \mathbf{k} \sigma}^{+}\left(c_{\lambda \mathbf{k} \sigma}\right)$ is the creation (annihilation) operator for a conduction electron with the wave vector $\mathbf{k}$, spin $\sigma$ in the lead $\lambda$ and $V_{\lambda \mathbf{k} \sigma}$ is the hybridization matrix element between localized electron on the dot with the energy $E_{d}$ and conduction electron of energy $\epsilon_{\lambda \mathbf{k}}$ in the lead $\lambda$.

The non-equilibrium Green's function technique [5] allows one to study the general situation with arbitrary value of the $\Delta T$. Here, however, we limit our consideration to the linear response. This means that the response coefficients $L_{i j}$ between charge and heat fluxes and chemical potential and temperature gradients are evaluated for an equilibrium $\left(\mu_{L}=\mu_{R}\right)$ system with constant temperature $\left(T_{L}=T_{R}\right)$. Electrical conductance $G$ is given by the coefficient $L_{11}$ as $G=\frac{-e^{2}}{T} L_{11}$, while thermopower requires knowledge of both $L_{11}$ and $L_{12}$ and is given by $S=\frac{-1}{e T} \frac{L_{12}}{L_{11}}$. The linear response coefficients read

$$
L_{11}=\frac{T}{h} \sum_{\sigma} \int d \omega \Gamma_{\sigma}(\omega) \operatorname{Im} G_{\sigma}^{r}(\omega)\left(-\frac{\partial f(\omega)}{\partial \omega}\right)_{T},
$$




$$
L_{12}=\frac{T^{2}}{h} \sum_{\sigma} \int d \omega \Gamma_{\sigma}(\omega) \operatorname{Im} G_{\sigma}^{r}(\omega)\left(\frac{\partial f(\omega)}{\partial T}\right)_{\mu} .
$$


Fig. 1. The linear response thermopower of the quantum dot coupled to magnetically polarised leads as a function of the position of the on-dot electron energy calculated for different temperatures (in units of $\Gamma$ ): $T=10^{-3}, T=10^{-2}$ and $T=10^{-1}$. Polarisation of the leads $P=0.0$ (upper panel) and $P=0.1$ (lower panel). Splitting of the Kondo resonance results in a very small thermopower.

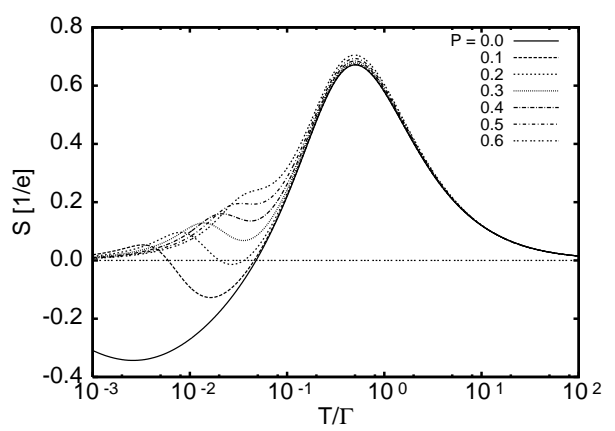

Fig. 2. The temperature dependence of the thermopower of the quantum dot coupled to magnetically polarised leads with $\mathrm{P}=0.0,0.1,0.2,0.3,0.4,0.5,0.6$ (starting from lowest curve near the deep minimum).

As it has already been noted in [6] in the context of N-QD-N system, the thermopower $\mathrm{S}$ is a powerful characteristic to identify the appearance of the Kondo effect as it changes sign when the system is cooled down to temperatures $T$ below $T_{K}$. This is particularly clear in Fig. 1 and in Fig. 2 (solid curve), where $\mathrm{S}$ vanishes for temperature equal actual Kondo temperature $T_{K}$. The behaviour is more complicated in the FM-QD-FM system with nonzero polarisation (lower panel of Fig. 1 and Fig. 2).

It is worth to note that the thermopower of the quantum dot defined in the two dimensional electron gas has recently been measured experimentally [7] and qualitatively agrees with that presented in figure 1 for $P=$ 0 . To understand their results the authors [7] use the Mott formula, which states that thermopower is proportional to the logarithmic derivative of the conductance with respect to the energy evaluated at the actual Fermi energy. The Kondo effect shows up as additional maximum appearing in conductance via quantum dot when the temperature decreases. It is connected with the Abrikosov-Suhl resonance (resonances) in the density of states, which appears (appear) at low temperatures and is (are) located at the position(s) of the Fermi level of the leads in (non)equilibrium situation. The slope of conductance thus changes and the thermopower changes sign. Thermopower measured directly and obtained from the Mott formula differ in line shapes [7] and this calls for the discussion of the validity of Mott formula [8] in the interacting quantum dot systems. This as well as detailed comparison between experimental data and calculations will be the subject of future work.

In magnetically polarised systems the Abrikosov Suhl resonance splits (as in external B field [2]) and this results in decrease of low temperature $\mathrm{S}$ (Fig. 1).

Acknowledgment This work has been supported by the Polish MNiI under the contract PBZ-MIN$\mathrm{P} 03 / 2003$.

\section{References}

[1] L. I. Glazman, M. E. Raikh, JETP Lett. 47, 452 (1988); T. K. Ng, P. A. Lee, Phys. Rev. Lett. 61, 1768 (1988).

[2] D. Goldhaber-Gordon et al., Nature 391, 158 (1998); S. M. Cronenwett et al., Science 281, 540 (1998).

[3] L.P. Kouwenhoven, L. Glazman, Physics World, Jan., 33 (2001).

[4] M. Krawiec, K. I. Wysokiński, Solid State Commun. 115, 141 (2000); Supercond. Sci. Technol. 17, 103 (2004).

[5] L. V. Keldysh, Sov. Phys. JETP 20, 10108 (1965); H. Haug, A. P. Yauho, Quantum Kinetics in Transport and Optics of Semiconductors, Springer, Berlin (1996).

[6] D. Boese, R. Fazio, Europhys. Lett. 56, 576 (2001); B. Dong, X. L. Lei, J. Phys.: Condens. Matter 14, 11747 (2002). 
[7] R. Scheibner et al. cond-mat/0410671 (2004).

[8] A. M. Lunde and K. Flensberg, J. Phys.: Condens. Matter 173879 (2005). 